\title{
Face recognition using selected topographical features
}

\author{
Maitham Ali Naji, Ghalib Ahmed Salman, Muthna Jasim Fadhil \\ College of Engineering and Electrical Technology, Middle Technical University (MTU), Iraq
}

\begin{tabular}{|c|c|}
\hline Article Info & ABSTRACT \\
\hline Article history: & This paper represents a new features selection method to improve an \\
\hline Received May 9, 2019 & $\begin{array}{l}\text { existed feature type. Topographical (TGH) features provide large set of } \\
\text { features by assigning each image pixel to the related feature depending }\end{array}$ \\
\hline Revised Mar 16, 2020 & on image gradient and Hessian matrix. Such type of features was \\
\hline Accepted Mar 28, 2020 & $\begin{array}{l}\text { handled by a proposed features selection method. A face recognition } \\
\text { feature selector (FRFS) method is presented to inspect TGH features. FRFS }\end{array}$ \\
\hline \multirow{6}{*}{$\begin{array}{l}\text { Keywords: } \\
\text { Face features } \\
\text { Face recognition } \\
\text { Feature selector } \\
\text { Features performance } \\
\text { topographical }\end{array}$} & $\begin{array}{l}\text { depends in its main concept on linear discriminant analysis (LDA) technique, } \\
\text { which is used in evaluating features efficiency. FRFS studies feature }\end{array}$ \\
\hline & behavior over a dataset of images to determine the level of its performance. \\
\hline & At the end, each feature is assigned to its related level of performance with \\
\hline & a chosen threshold, the highest set of features is selected to be classified by \\
\hline & SVM classifier. \\
\hline & $\begin{array}{r}\text { Copyright } \odot 2020 \text { Institute of Advanced Engineering and Science. } \\
\text { All rights reserved. }\end{array}$ \\
\hline
\end{tabular}

\section{Corresponding Author:}

Ghalib Ahmed Salman,

College of Engineering and Electrical Technology,

Middle Technical University (MTU),

Al-Masafee Street, Al-Doora, Baghdad, Iraq.

Email: ghlbethawi@gmail.com

\section{INTRODUCTION}

Face recognition was deeply studied through the last decades [1]. Several types of features were presented with different levels of recognition accuracy [2]. Each type of feature represent an amount of information extracted from the image, and more represented information in chosen feature provides more accuracy in recognition results [3]. Spatial filtering was adopted to extract points of interest, which are grouped to form candidate features. A probability-based statistical model is built to select the most powerful features for face recognition [4]. In another approach, the authors used RGB-D images based on the extracting and the concatenating of the scale invariant feature transform (SIFT) descriptors from these data sources for face recognition [5]. Jie Chen et al turned transformation feature by adopting specific version of local binary patterns (LBP) as a face descriptor. Such features provided encouraging classification accuracy, but with unacceptable error rate sometimes [6]. Face descriptor is also adopted for extracting face feature in order to provide face recognition. The authors adopted enhanced local binary pattern (EnLBP) as an adaption of LBP and they divided studied image into $3 \times 3$ blocks. For each block, the EnLBP features provided mean values of each block instead of the ordinary number of LBP [7].

In this paper, since TGH provide more information than traditional edges [8], they are adopted by combining them with the proposed feature selection method to choose the best set among them. The rest of this of this paper contains brief explanation of TGH then the selection method; then face recognition results and their analysis. Final section contains the conclusions extracted from this work.

\section{TOPOGRAPHICAL FEATURES TGH}

Although TGH were previously used in recognition researches [9, 10], their accuracy results were relatively low regarding other types of features. One of the potential reasons of the lack in their usage is the huge amount of produce features. Recently, it was used in object recognition researches but with the same 
problem of huge number of features [11]. Since TGH assigns each image pixel to the corresponding feature. On the other side, TGH provides more information than traditional image edges and lines, see Figure 1(a). Yet, and choosing only image lines and edges may ignore significant information.

Such problem can be solved by choosing efficient ones among produced features overall image, which was a noticeable obstacle [12]. After manipulating the image with set of masks to find the orthogonal polynomial of the image [13], image gradient and Hessian matrix of second derivatives are computed to extract TGH (Peak, Pit, Saddle, Ridge, Ravine, Zero crossing, Flat, Increasing area and Decreasing area) [14], see Figure 1(b). According to definitions, each literature defines some kinds of TGH to be image edges and lines. As a result, dealing with TGH provides more information, and the next section explains the proposed feature selection method to overcome high number of provided feature. Since TGH is an existed feature type, this paper focuses on explaining feature selection, and aforementioned literatures discuss TGH in details.

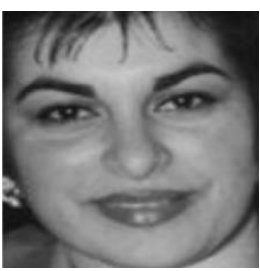

(a)

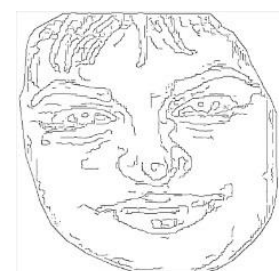

(b)

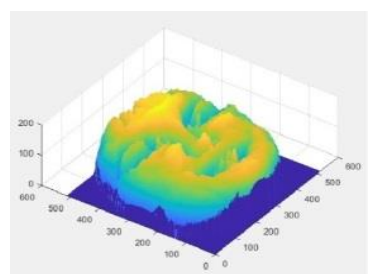

(c)

Figure 1. Traditional image edges ignore significant information, while TGH study all available features: (a) original image, (b) image edges, and (c) TGH fFeatures

\section{FACE RECOGNITION FEATURE SELECTOR (FRFS)}

This method seeks for features using similar rules used in LDA to evaluate feature significance [15]. According to non-parametric feature test in LDA, efficient features must yield high differences over inter-class images against low differences over intra-class images [16]. This technique adopt testing single feature in each round by measuring yielded differences over inter and intra-class to record its level of efficiency. Then proposed technique moves to the second feature and so on till the last feature. Different levels of efficiency are filtered by controlled threshold that determines selected set of features with highest efficiency. Locations of selected features from learning dataset are allocated to choose recognition features from test dataset in test stage.

To illustrate proposed FRFS, assume that learning dataset contains $n \times m$ images contain $n$ persons and $\mathrm{m}$ image for each of them. Each the image $\mathrm{Im}_{\mathrm{ij}} \mathrm{is}$ of $\mathrm{h} \times \mathrm{k}$ size, which is used to produce TGH features as:

$$
i m_{i j}=\left[\begin{array}{lllll}
f_{11} & f_{12} & f_{13} & \cdots & f_{1 k} \\
f_{21} & f_{22} & f_{23} & \cdots & f_{2 k} \\
f_{h 1} & f_{h 2} & f_{h 3} & \cdots & f_{h k}
\end{array}\right]
$$

The first feature from each image in learning dataset is transferred to the inspection matrix:

$$
I_{11}=\left[\begin{array}{lllll}
\mathrm{f} 11_{11} & \ldots & \mathrm{f} 11_{1 \mathrm{j}} & \ldots & \mathrm{f} 11_{1 \mathrm{~m}} \\
\mathrm{f} 11_{\mathrm{i} 1} & \ldots & \mathrm{f} 11_{\mathrm{ij}} & \ldots & \mathrm{f} 11_{\mathrm{im}} \\
\mathrm{f} 11_{\mathrm{n} 1} & \ldots & \mathrm{f} 11_{\mathrm{nj}} & \ldots & \mathrm{f} 11_{\mathrm{nm}}
\end{array}\right]
$$

where $I_{11}$ : is the inspection matrix of the feature $f_{11}$ over learning dataset

$f 11_{i j}$ : the first feature f11 taken from the image in the row $i$ and column $j$ of the learning dataset

Statistical laws dispersion are used to measure the changes within values over inter-class and intra-class, which are standard deviation SD and roughness coefficient $r c$ [17]. Values dispersion is measuring using SD by computing their deviation from the average, and it is usedin this paper to measure two types of changes. Firstly, SD over each row is used to evaluate the changes over different images for the same person, which represents interior differences (intra-changes). Secondly, SD over each column is used to evaluate changes over different person in each line of images (inter-changes). The $\mu$ and SD of each row are computed as:

$$
\mu_{i}=\frac{\sum_{j=1}^{m} f 11_{i j}}{m}
$$




$$
\mathrm{SD}_{\mathrm{ri}}=\frac{\sum_{\mathrm{j}=1}^{\mathrm{m}}\left(\mathrm{f} 11_{\mathrm{ij}}-\mu_{\mathrm{i}}\right)^{2}}{\mathrm{~m}}
$$

Accordingly, $\mu$ and SD of each column are computed as:

$$
\begin{aligned}
& \mu_{j}=\frac{\sum_{i=1}^{n} f 11_{i j}}{n} \\
& \mathrm{SD}_{c j}=\frac{\sum_{i=1}^{n}\left(f 11_{i j}-\mu_{j}\right)^{2}}{n}
\end{aligned}
$$

This produces the next matrix:

$$
\mathrm{I}_{11}=\left[\begin{array}{cccccc}
\mathrm{f} 11_{11} & \ldots & \mathrm{f} 11_{1 \mathrm{j}} & \ldots & \mathrm{f} 11_{1 \mathrm{~m}} & \mathrm{SD}_{\mathrm{r} 1} \\
\mathrm{f} 11_{\mathrm{i} 1} & \ldots & \mathrm{f} 11_{\mathrm{ij}} & \ldots & \mathrm{f} 11_{\mathrm{im}} & \mathrm{SD} \\
\mathrm{f} 11_{\mathrm{n} 1} & \cdots & \mathrm{f} 11_{\mathrm{nj}} & \ldots & \mathrm{f} 11_{\mathrm{nm}} & \mathrm{SD} \\
\mathrm{SD}_{\mathrm{c} 1} & \cdots & \mathrm{SD}_{\mathrm{cj}} & \cdots & \mathrm{SD}_{\mathrm{cm}} &
\end{array}\right]
$$

SD for each row is called $S D_{r i}$ to distinguish them from SD for each column $\left(S D_{c i}\right)$. Several values of SD are produced over intra-changes one for each person. To get one SD value over all intra-class, $S D_{\text {intra }}$ is computed using next average rule:

$$
\mathrm{SD}_{\text {intra }}=\frac{\sum_{\mathrm{i}=1}^{\mathrm{n}} \mathrm{SD}_{\mathrm{ri}}}{\mathrm{n}}
$$

Accordingly, $\mathrm{SD}_{\text {inter }}$ is computed as:

$$
\mathrm{SD}_{\text {inter }}=\frac{\sum_{\mathrm{j}=1}^{\mathrm{m}} \mathrm{SD}_{\mathrm{cj}}}{\mathrm{m}}
$$

The final value of efficiencyE $\mathrm{E}_{\text {final }}$ for the feature $f_{11}$ is computed by dividing $\mathrm{SD}_{\text {inter }}$ over $\mathrm{SD}_{\text {intra }}$ as:

$$
E_{\text {final }}=\frac{S D_{\text {inter }}}{S D_{\text {intra }}}
$$

The second measure of dispersion is roughness coefficient which divides the differences between the successive values over their differences from the mean. Such measure reduces or eliminates the effect of big differences between successive values [17].

$$
\mathrm{rc}=\frac{\sum_{\mathrm{i}=2}^{\mathrm{n}}\left(\mathrm{x}_{\mathrm{i}}-\mathrm{x}_{\mathrm{i}-1}\right)^{2}}{\sum_{\mathrm{i}=2}^{\mathrm{n}}\left(\mathrm{x}_{\mathrm{i}}-\mu\right)^{2}}
$$

In some cases, successive images yield significant differences due to different styles, face hair, face expression or other factors, which can be handled using $r c$. Accordingly, the matrix in (7) will be:

$$
\mathrm{I}_{11}=\left[\begin{array}{cccccc}
\mathrm{f} 11_{11} & \ldots & \mathrm{f} 11_{1 \mathrm{j}} & \ldots & \mathrm{f} 11_{1 \mathrm{~m}} & \mathrm{rc}_{\mathrm{r} 1} \\
\mathrm{f} 11_{\mathrm{i} 1} & \ldots & \mathrm{f} 11_{\mathrm{ij}} & \ldots & \mathrm{f} 11_{\mathrm{im}} & \mathrm{rc} \\
\mathrm{f} 11_{\mathrm{ri}} & \cdots & \mathrm{f} 11_{\mathrm{nj}} & \ldots & \mathrm{f} 11_{\mathrm{nm}} & \mathrm{r} \\
\mathrm{rc}_{\mathrm{c} 1} & \cdots & \mathrm{rc}_{\mathrm{cj}} & \cdots & \mathrm{rc}_{\mathrm{cm}} & \mathrm{rr}
\end{array}\right]
$$

The best value of $\mathrm{E}_{\text {final }}$ is the biggest one since it refers $\mathrm{SD}_{\text {inter }}$ has highest level regarding $\mathrm{SD}_{\text {intra }}$. $\mathrm{E}_{\text {final }}$ is transferred to the corresponding location in a matrix for Efficiency of Features $E F F$, thus, $\mathrm{E}_{\text {final }}$ for the feature $f_{11}$ is transferred to $E F F(1,1)$. Equations (2) to (10) are re-applied on the next feature and so on until covering all features in the image matrix $i m_{i j}$. As result, $E F F$ matrix will contain different values for different levels of efficiency of features, and choosing features with highest efficiency provide best set of candidate features. To control the number of chosen features, a threshold is proposed, and values of $\mathrm{E}_{\text {final }}$ lower than the threshold is ignored. Depending on chosen set of features, images are classified using the standard support vector machine SVM $[18,19]$. 


\section{RESULTS AND DISCUSSION}

Experiments of this work used fairly distorted and good images of standard FG-NET which contains (912 of 1002) face-images, which are captured for males and females in different occasions. Highly distorted images are excluded from the study due to their affection on yielded results. All studied images are manipulated to be gray-scale and with size of $(512 \times 512)$. Comparison used in this paper is mean absolute error (MAE).

Although building 2D polynomial for the image uses set of enhancing masks, image quality affects recognition rate (RR). Low image quality may cause blurry or add lines and undesirable details to the image, which may cause non-real details. Where TGH depends on differences between values [20], undesirable effects may create non-real features as shown in Figure 2.

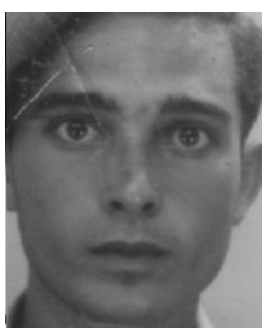

(a)

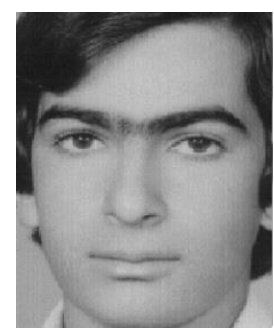

(b)

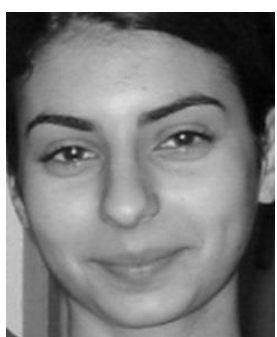

(c)

Figure 2. Image quality affects the classification accuracy and causes error rate in recognition results:

(a) fairly distorted MAE=92.78, (b) acceptable MAE=93.93, and (c) high quality MAE=94.12

As face recognition feature selector (FRFS) measures the performance of each feature and chooses the highest set of them using a threshold, controlling the threshold value affects RR value. Increasing the threshold value provides features with highest level of performance, yet it may ignore significant set of features, and big number of chosen features requires longer processing time. On the other side, decreasing threshold value ensures choosing all significant features, but they be combined by low level-performance features, which affects recognitions accuracy. Experiments yielded best results when threshold value chooses the highest $27 \%$ of the features performance. Only low percentage of features is chosen, though such percentage provides significant number of features due to the huge number of produced features. See Figure 3.

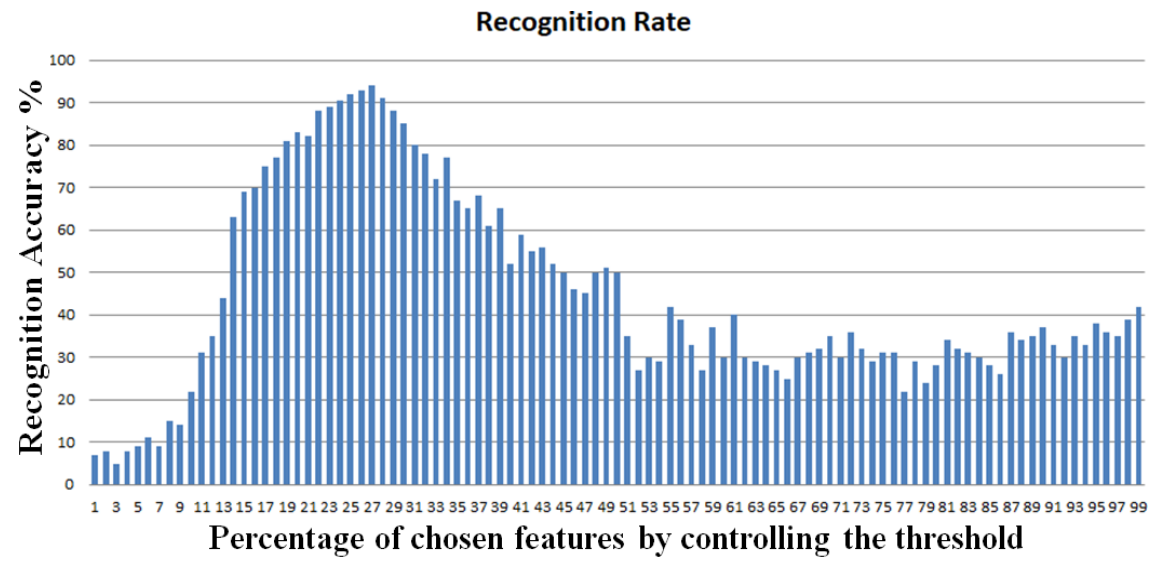

Figure 3. Recognition rate due to the value of chosen threshold

FG-Net face dataset is collected from number of ordinary images, which are not prepared for scientific purposes [21]. Faces in such images may contain glasses, hair style and face hair as shown in Figure 4. Such undesirable conditions affects recognition accuracy, yet experiments yielded encouraging results as shown in Table 1. Faces in some of images are rotated in two directions, first one is the rotation around the vertical axis when some persons are leaning his head to the left or right, while some others turns their faces to left or right direction around the horizontal axis as shown in Figure 5. 

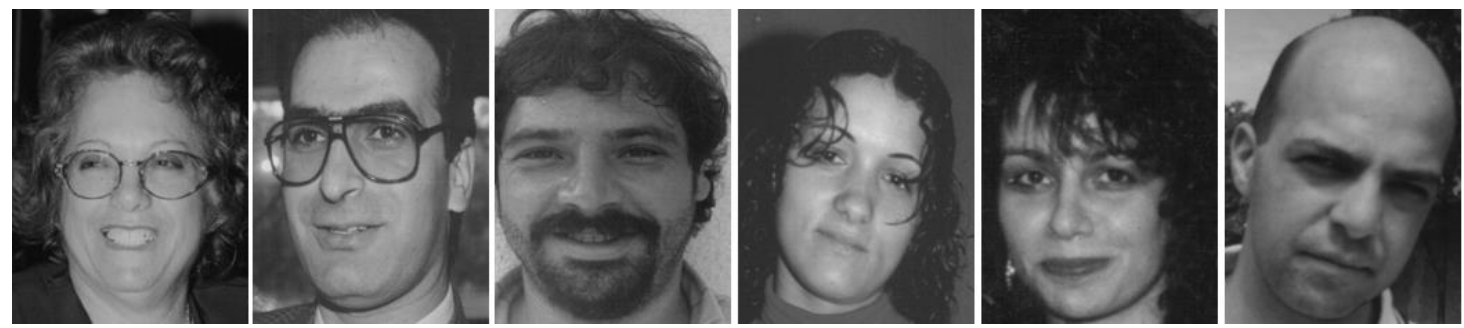

Figure 4. Glasses, hair style and face hair affect the recognition by hiding some features or creating new ones

Table 1. Recognition rate under the effects of glasses, hair style and face hair

\begin{tabular}{cc}
\hline Condition & RR \\
\hline Hair Style & 93.41 \\
Face Hair & 93.03 \\
Glasses & 92.81 \\
\hline
\end{tabular}
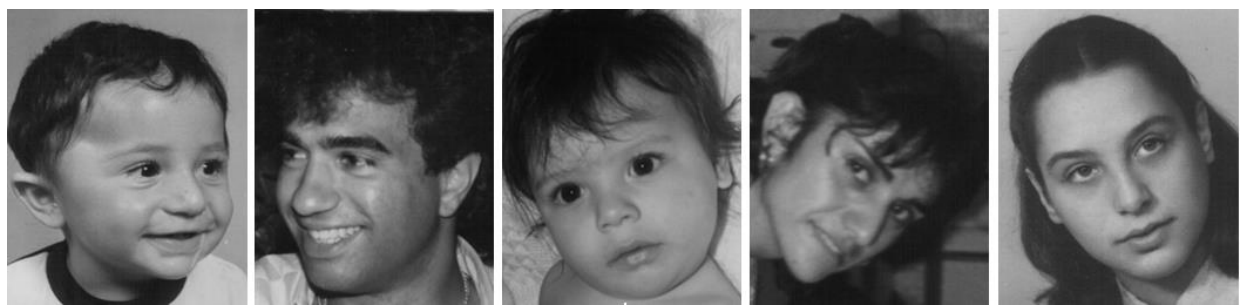

Figure 5. Some faces are rotated around the horizontal or vertical axis

Experimental results showed that $\mathrm{TGH}$ features have more robustness against rotation around the vertical axis, which is justified by two points. As TGH features are produced using differences between values rather than the values themselves, they have lower level of effects against rotation and illumination [9]. Secondly, this type of rotation changes features positions only, while the second type of rotation hides or creates features due to the illumination and the view angle. Rotation around the horizontal axis yielded lower rate in recognition accuracy.

For benchmarking with state of art, proposed collection between TGH features and FRFS method yielded encouraging results. Comparing with highest yielded RR, proposed technique yielded noticeable improvement considering all types of tested images by reaching (93.95\%) for recognition accuracy, while best results are yielded by applying proposed technique on images with best condition when TGH and FRFS reach (94.12) for recognition accuracy as shown in Table 2.

Table 2. Comparing proposed technique with state of art

\begin{tabular}{lr}
\hline \multicolumn{1}{c}{ Techniques } & RR \\
\hline Affine transformation due to viewing angle and distance variations. Therefore, affine invariant feature extraction [22] & 92.50 \\
Local and Holistic features with Neural Networks [23] & 93.46 \\
Signal Reconstruction with Neural Networks[24] & 93.89 \\
HAAR-like features and eigenfaces [25] & 93.91 \\
Proposed Technique (Average) & $\mathbf{9 3 . 9 5}$ \\
Proposed Technique (Best) & $\mathbf{9 4 . 1 2}$ \\
\hline
\end{tabular}

\section{CONCLUSION}

In this paper, a collection of topographical features which are extracted from face images after finding the $2 \mathrm{D}$ polynomial of the image. They are extracted by computing image gradient and Hessian matrix of second order derivatives of image. Due the huge number of produced features, the proposed technique of face recognition feature selection FRFS is applied to choose the best set of produced features. Selected features recorded robustness against rotation with insignificant level of effects for glasses, hair style and face hair. The entire performance of the proposed technique recorded encouraging results against state of art. For future works, we propose studying the effects of facial expression on TGH features extracted by FRFS. 


\section{REFERENCES}

[1] M. M. Kasar, D. Bhattacharyya, and T. H. Kim, "Face recognition using neural network: a review," International Journal of Security and Its Applications, vol. 10, no. 3, pp. 81-100, 2016.

[2] R. Rouhi, et al, "A Review on Feature Extraction Techniques in Face Recognition," Signal \& Image Processing: An International Journal (SIPIJ), vol. 3, no. 6, 2012.

[3] I. Guyon, et al, "Feature Extraction, Foundations and Applications," Springer, 2006.

[4] K. C. Yow and R. Cipolla, "Feature-based Human Face Detection," Journal of Image and Vision Computing, vol. 15 , no. 9, pp. 713-735, 1997.

[5] H. Ouanan, M. Ouanan, and B. Aksasse, "Gabor-HOG features based face recognition scheme," TELKOMNIKA Indonesian Journal of Electrical Engineering, vol. 15, no. 2, pp. 331-335, 2015.

[6] J. Chen et al, "Robust local features for remote face recognition," Journal of Image and Vision Computing, vol. 64, pp. 34-46, 2017.

[7] H. H. Abbas, A. A. Altameemi, and H. R. Farhan, "Biological landmark vs quasi-landmarks for 3D face recognition and gender classification," International Journal of Electrical and Computer Engineering (IJECE), vol. 9, no. 5, pp. 4069-4076, 2019.

[8] M. E. Wibowo, et al., "Improved Face Recognition across Poses using Fusion of Probabilistic Latent Variable Models," TELKOMNIKA Telecommunication Computing Electronics and Control, vol. 15, no. 4, pp. 1971-1981, 2017.

[9] L. Wang and T. Pavlidis, "Direct Gray-Scale Extraction of Features for Character recognition," IEEE Transactions on Pattern Analysis and Machine Intelligence, vol. 15, no. 10, pp. 1053-1067, 1993.

[10] S.-W. Lee and Y. J. Kim, "Direct Extraction of Topographic Features for Gray Scale Character Recognition," IEEE Transactions on Pattern Analysis and Machine Intelligence, vol. 17, no. 7, pp. 724-728, 1995.

[11] N. S. Rani, S. K. Verma, and A. Joseph, "A Zone Based Approach for Classification and Recognition of Telugu Handwritten Characters," International Journal of Electrical and Computer Engineering (IJECE), vol. 6, no. 4, pp. 1647-1653, 2016.

[12] P. VenkateswarLal, G. R. Nitta, and A. Prasad, "Ensemble of texture and shape descriptors using support vector machine classification for face recognition" Journal of Ambient Intelligence and Humanized Computing, pp. 1-8, 2019.

[13] E. B. Moody, "Discrete Orthogonal Polynomial Restoration of Images Degraded by Spatially Varying Point Spread Functions," Proceedings of 1st International Conference on Image Processing, pp. 680-684, 1994.

[14] R. M. Haralick, "Digital Step Edges from Zero Crossing of Second Directional Derivatives," IEEE Transactions on Pattern Analysis and Machine Intelligence, vol. 1, pp. 58-68, 1984.

[15] W. Chen, M. J. Er, and S. Wu, "PCA and LDA in DCT Domain," Pattern Recognition Letters, vol. 26, no. 15, pp. 2474-2482, 2005.

[16] I. G. P. S. Wijaya, A. Y. Husodo, and I. W. A. Arimbawa, "Real Time Face Recognition Based on Face Descriptor and Its Application," TELKOMNIKA Telecommunication Computing Electronics and Control, vol. 16, no. 2, pp. 739-746, 2018.

[17] R. V. Hogg, J. McKean, and A. T. Craig, "Introduction to mathematical statistics," Pearson Education, 2005.

[18] J. A. Suykens and J. Vandewalle, "Least squares support vector machine classifiers," Neural processing letters, vol. 9, no. 3, pp. 293-300, 1999.

[19] F. E. Gunawan, J. Harefa, and N. Sekishita, "Face Recognition on Linear Motion-blurred Image," TELKOMNIKA (Telecommunication Computing Electronics and Control), vol. 16, no. 3, pp. 1249-1255, 2018.

[20] S. Mizugaki, et al., "Fingerprinting suspended sediment sources in the Nukabira River, northern Japan," International Journal of Erosion Control Engineering, vol. 5, no. 1, pp. 60-69, 2012.

[21] R. P. Persada, et al., "Automatic face and VLP'S recognition for smart parking system," TELKOMNIKA (Telecommunication Computing Electronics and Control), vol. 17, no. 4, pp. 1698-1705, 2019.

[22] L. Yu, et al., "Affine invariant fusion feature extraction based on geometry descriptor and BIT for object recognition," IET Image Processing, vol. 13, no. 1, pp. 57-72, 2018.

[23] S. Xie and H. Hu, "Facial Expression Recognition Using Hierarchical Features With Deep Comprehensive Multipatches Aggregation Convolutional Neural Networks," IEEE Transactions on Multimedia, vol. 21, no. 1, pp. 211-220, 2019.

[24] B. Xu, Q. Liu, and T. Huang, "A discrete-time projection neural network for sparse signal reconstruction with application to face recognition," IEEE transactions on neural networks and learning systems, vol. 30, no. 1, pp. 151-162, 2018.

[25] G. I. Hapsari, G. A. Mutiara, and H. Tarigan, "Face recognition smart cane using HAAR-like features and eigenfaces," TELKOMNIKA Telecommunication Computing Electronics and Control, vol. 17, no. 2, pp. 973-980, 2019. 